\title{
A wide-spectrum language for verification of programs on weak memory models
}

\author{
Robert J. Colvin and Graeme Smith \\ School of Information Technology and Electrical Engineering \\ University of Queensland
}

\begin{abstract}
Modern processors deploy a variety of weak memory models, which for efficiency reasons may (appear to) execute instructions in an order different to that specified by the program text. The consequences of instruction reordering can be complex and subtle, and can impact on ensuring correctness. Previous work on the semantics of weak memory models has focussed on the behaviour of assembler-level programs. In this paper we utilise that work to extract some general principles underlying instruction reordering, and apply those principles to a widespectrum language encompassing abstract data types as well as low-level assembler code. The goal is to support reasoning about implementations of data structures for modern processors with respect to an abstract specification.

Specifically, we define an operational semantics, from which we derive some properties of program refinement, and encode the semantics in the rewriting engine Maude as a model-checking tool. The tool is used to validate the semantics against the behaviour of a set of litmus tests (small assembler programs) run on hardware, and also to model check implementations of data structures from the literature against their abstract specifications.
\end{abstract}

\section{Introduction}

Modern processor architectures provide a challenge for developing efficient and correct software. Performance can be improved by parallelising computation to utilise multiple cores, but communication between threads is notoriously errorprone. Weak memory models go further and improve overall system efficiency through sophisticated techniques for batching reads and writes to the same variables and to and from the same processors. However, code that is run on such memory models is not guaranteed to take effect in the order specified in the program code, creating unexpected behaviours for those who are not forewarned [1. For instance, the instructions $x:=1 ; y:=1$ may be reordered to $y:=1 ; x:=1$. Architectures typically provide memory barrier/fence instructions which can enforce local ordering - so that $x:=1$; fence ; $y:=1$ can not be reordered - but reduce performance improvements (and so should not be overused).

Previous work on formalising weak memory models has resulted in abstract formalisations which were developed incrementally through communication with 
processor vendors and rigorous testing on real machines 22139]. A large collection of "litmus tests" have been developed [216] which demonstrate the sometimes confusing behaviour of hardware. We utilise this existing work to provide a wide-spectrum programming language and semantics that runs on the same relaxed principles that apply to assembler instructions. When these principles are specialised to the assembler of ARM and POWER processors our semantics gives behaviour consistent with existing litmus tests. Our language and semantics, therefore, connect instruction reordering to higher-level notions of correctness. This enables verification of low-level code targeting specific processors against abstract specifications.

We begin in Sect. 2 with the basis of an operational semantics that allows reordering of instructions according to pair-wise relationships between instructions. In Sect. 3 we describe the semantics in more detail, focussing on its instantiation for the widely used ARM and POWER processors. In Sect. 4 we give a summary of the encoding of the semantics in Maude and its application to model-checking concurrent data structures. We discuss related work in Sect. 5 before concluding in Sect. 6.

\section{Instruction reordering in weak memory models}

\section{$2.1 \quad$ Thread-local reorderings}

It is typically assumed processes are executed in a fixed sequential order (as given by sequential composition - the "program order"). However program order may be inefficient, e.g., when retrieving the value of a variable from main memory after setting its value, as in $\mathrm{x}:=1 ; \mathrm{r}:=\mathrm{x}$, and hence weak memory models sometimes allow execution out of program order to improve overall system efficiency. While many reorderings can seem surprising, there are basic principles at play which limit the number of possible permutations, the key being that the new ordering of instructions preserves the original sequential intention.

A classic example of weak memory models producing unexpected behaviour is the "store buffer" pattern below [2]. Assume that all variables are initially 0 , and that thread-local variables (registers) are named $r, r_{1}, r_{2}$, etc., and that $x$ and $y$ are shared variables.

$$
\left(x:=1 ; r_{1}:=y\right) \|\left(y:=1 ; r_{2}:=x\right)
$$

It is possible to reach a final state in which $r_{1}=r_{2}=0$ in several weak memory models: the two assignments in each process are independent (they reference different variables), and hence can be reordered. From a sequential semantics perspective, reordering the assignments in process 1 , for example, preserves the final values for $r_{1}$ and $x$.

Assume that $c$ and $c^{\prime}$ are programs represented as sequences of atomic actions $\alpha ; \beta ; \ldots$, as in a sequence of instructions in a processor or more abstractly a semantic trace. Program $c$ may be reordered to $c^{\prime}$, written $c \leadsto c^{\prime}$, if the following holds: 
1. $c^{\prime}$ is a permutation of the actions of $c$, possibly with some modifications due to forwarding (see below).

2. $c^{\prime}$ preserves the sequential semantics of $c$. For example, in a weakest preconditions semantics [8], $\left(\forall S \bullet w p(c, S) \Rightarrow w p\left(c^{\prime}, S\right)\right)$.

3. $c^{\prime}$ preserves coherence-per-location with respect to $c$ (cf. po-loc in [3]). This means that the order of updates and accesses of each shared variable, considered individually, is maintained.

We formalise these constraints below. The key challenge for reasoning about programs executed on a weak memory model is that the behaviour of $c \| d$ is in general quite different to the behaviour of $c^{\prime} \| d$, even if $c \sim c^{\prime}$.

\subsection{Reordering and forwarding instructions}

We write $\alpha \stackrel{\text { R }}{=} \beta$ if instruction $\beta$ may be reordered before instruction $\alpha$. It is relatively straightforward to define when two assignment instructions (encompassing stores, loads, and register operations at the assembler level) may be reordered. Below let $x$ nfi $f$ mean that $x$ does not appear free in the expression $f$, and say expressions $e$ and $f$ are load-distinct if they do not reference any common shared variables.

$$
x:=e \stackrel{\text { R }}{=} y:=f \quad \text { if } \quad \begin{aligned}
& \text { 1) } x, y \text { are distinct; 2) } x \text { nfi } f \text {; 3) } y \text { nfi } e \text {; and } \\
& \text { 4) } e, f \text { are load-distinct; }
\end{aligned}
$$

Note that in general $\stackrel{R}{=}$ is not reflexive: in TSO processors a load may be reordered before a store, but not vice versa 23 .

Provisos 1), 2) and 3) ensure executing the two assignments in either order results in the same final values for $x$ and $y$, and proviso 4) maintains order on accesses of the shared state. If two updates do not refer to any common variables they may be reordered. The provisos allow some reordering when they share common variables. Proviso 1) eliminates reorderings such as $(x:=1 ; x:=2) \sim$ $(x:=2 ; x:=1)$ which would violate the sequential semantics (the final value of $x)$. Proviso 2) eliminates reorderings such as $(x:=1 ; r:=x) \sim(r:=x ; x:=1)$ which again would violate the sequential semantics (the final value of $r$ ). Proviso $3)$ eliminates reorderings such as $(r:=y ; y:=1) \leadsto(y:=1 ; r:=y)$ which again would violate the sequential semantics (the final value of $r$ ). Proviso 4), requiring the update expressions to be load-distinct, preserves coherence-perlocation, eliminating reorderings such as $\left(r_{1}:=x ; r_{2}:=x\right) \leadsto\left(r_{2}:=x ; r_{1}:=x\right)$, where $r_{2}$ may receive an earlier value of $x$ than $r_{1}$ in an environment which modifies $x$.

In practice, proviso 2) may be circumvented by forwarding 1 . This refers to taking into account the effect of the earlier update on the expression of the latter. We write $\beta_{[\alpha]}$ to represent the effect of forwarding the (assignment) instruction $\alpha$ to the instruction $\beta$. For assignments we define

$$
(y:=f)_{[x:=e]}=y:=\left(f_{[x \backslash e]}\right) \quad \text { if } \quad e \text { does not refer to global variables }
$$

\footnotetext{
${ }^{1}$ We adopt the term "forwarding" from ARM and POWER [3]. The equivalent effect is referred to as bypassing on TSO [23].
} 
where the term $f_{[x \backslash e]}$ stands for the syntactic replacement in expression $f$ of references to $x$ with $e$. The proviso of (3) prevents additional loads of globals being introduced by forwarding.

We specify the reordering and forwarding relationships with other instructions such as branches and fences in Sect. 3.3.

\subsection{General operational rules for reordering}

The key operational principle allowing reordering is given by the following transition rules for a program $(\alpha ; c)$, i.e., a program with initial instruction $\alpha$.

$$
(\alpha ; c) \stackrel{\alpha}{\longrightarrow} c \quad(a) \quad \frac{c \stackrel{\beta}{\longrightarrow} c^{\prime} \alpha \stackrel{R}{\models} \beta_{[\alpha]}}{(\alpha ; c) \stackrel{\beta_{[\alpha]}}{\longrightarrow}\left(\alpha ; c^{\prime}\right)}
$$

Rule (4a) is the straightforward promotion of the first instruction into a step in a trace, similar to the basic prefixing rules of CCS [18] and CSP [11]. Rule (44), however, states that, unique to weak memory models, an instruction of $c$, say $\beta$, can happen before $\alpha$, provided that $\beta_{[\alpha]}$ can be reordered before $\alpha$ according to the rules of the architecture. Note that we forward the effect of $\alpha$ to $\beta$ before deciding if the reordering is possible.

Applying Rule (40) then Rule (4a) gives the following reordered behaviour of two assignments.

$$
(r:=1 ; x:=r ; \text { nil }) \stackrel{x:=1}{\longrightarrow}(r:=1 ; \text { nil }) \stackrel{r:=1}{\longrightarrow} \text { nil }
$$

We use the command nil to denote termination. The first transition above is possible because we calculate the effect of $r:=1$ on the update of $x$ before executing that update, i.e., $x:=r_{[r:=1]}=x:=1$.

The definitions of instruction reordering, $\alpha \stackrel{\text { R }}{=} \beta$, and instruction forwarding, $\beta_{[\alpha]}$ are architecture-specific, and are the only definitions required to specify an architecture's instruction ordering 2 The instantiations for sequentially consistent processors (i.e., those which do not have a weak memory model) are trivial: $\alpha F^{R} \beta$ for all $\alpha, \beta$, and there is no forwarding. Since reordering is not possible Rule (44) never applies and hence the standard prefixing semantics is maintained. TSO is relatively straightforward: loads may be reordered before stores (provided they reference different shared variables). In this paper we focus on the more complex ARM and POWER memory models. These memory models are very similar, the notable difference being the inclusion of the lightweight fence instruction in POWER. Due to space limitations, we omit lightweight fences in this paper but a full definition which has been validated against litmus tests can be found in Appendix A

\footnotetext{
${ }^{2}$ Different architectures may have different storage subsystems, however, and these need to be separately defined (see Sect. 3.2).
} 


\subsection{Reasoning about reorderings}

The operational rules allow a standard trace model of correctness to be adopted, that is, we say programs $c$ refines to program $d$, written $c \sqsubseteq d$, iff every trace of $d$ is a trace of $c$. Let the program $\alpha \cdot c$ have the standard semantics of prefixing, that is, the action $\alpha$ always occurs before any action in $c$ (Rule (4a)). Then we can derive the following laws that show the interplay of reordering and true prefixing.

$$
\begin{aligned}
\alpha ; c & \sqsubseteq \alpha \cdot c \\
\alpha ;(\beta \cdot c) & \sqsubseteq \beta_{[\alpha]} \cdot(\alpha ; c) \quad \text { if } \alpha \stackrel{\text { R }}{\models} \beta_{[\alpha]}
\end{aligned}
$$

Note that in Law (7) $\alpha$ may be further reordered with instructions in $c$. A typical interleaving law is the following.

$$
(\alpha \cdot c) \| d \sqsubseteq \alpha \cdot(c \| d)
$$

We may use these laws to show how the "surprise" behaviour of the store buffer pattern above arises 3 In derivations such as the following, to save space, we abbreviate a thread $\alpha$; nil or $\alpha$. nil to $\alpha$, that is, we omit the trailing nil.

$$
\begin{aligned}
& \left(x:=1 ; r_{1}:=y\right) \|\left(y:=1 ; r_{2}:=x\right) \\
& \sqsubseteq \quad \text { From Law (7) (twice), since } x:=1 \stackrel{\text { R }}{\models} r_{1}:=y \text { from (2). } \\
& \left(r_{1}:=y \cdot x:=1\right) \|\left(r_{2}:=x \cdot y:=1\right) \\
& \sqsubseteq \text { Law (8) (four times) and commutativity of } \| \text {. } \\
& r_{1}:=y \cdot r_{2}:=x \cdot x:=1 \cdot y:=1
\end{aligned}
$$

If initially $x=y=0$, a standard sequential semantics shows that $r_{1}=r_{2}=0$ is a possible final state in this behaviour.

\section{Semantics}

\subsection{Formal language}

The elements of our wide-spectrum language are actions (instructions) $\alpha$, commands (programs) $c$, processes (local state and a command) $p$, and the top level system $s$, encompassing a shared state and all processes. Below $x$ is a variable (shared or local) and $e$ an expression.

$$
\begin{aligned}
& \alpha:=x:=e \quad \mid \quad[e] \quad \text { fence } \mid \text { cfence } \mid \alpha^{*} \\
& c::=\operatorname{nil}|\alpha ; c| c_{1} \sqcap c_{2} \mid \text { while } b \text { do } c \\
& p::=(\operatorname{lcl} \sigma \bullet c)\left|\left(\operatorname{tid}_{\mathrm{N}} p\right)\right| p_{1} \| p_{2} \\
& s::=(\operatorname{glb} \sigma \bullet p) \mid(\operatorname{stg} W \bullet p)
\end{aligned}
$$

3 To focus on instruction reorderings we leave local variable declarations and process ids implicit, and assume a multi-copy atomic storage system (see Sect. 3.2). 
An action may be an update $x:=e$, a guard [e], a (full) fence, a control fence (see Sect. 3.3), or a finite sequence of actions, $\alpha^{*}$, executed atomically. Throughout the paper we denote an empty sequence by \langle\rangle , and construct a non-empty sequence as $\left\langle\alpha_{1}, \alpha_{2} \ldots\right\rangle$.

A command may be the empty command nil, which is already terminated, a command prefixed by some action $\alpha$, a choice between two commands, or an iteration (for brevity we consider only one type of iteration, the while loop). Conditionals are modelled using guards and choice.

$$
\text { if } b \text { then } c_{1} \text { else } c_{2} \widehat{=}\left([b] ; c_{1}\right) \sqcap\left([\neg b] ; c_{2}\right)
$$

A well-formed process is structured as a process id $\mathrm{N} \in \mathrm{PID}$ encompassing a (possibly empty) local state $\sigma$ and command $c$, i.e., a term $\left(\operatorname{tid}_{\mathrm{N}} \mathbf{l c l} \sigma \bullet c\right)$. We assume that all local variables referenced in $c$ are contained in the domain of $\sigma$.

A system is structured as the parallel composition of processes within the global storage system, which may be either a typical global state, $\sigma$, that maps all global variables to their values (modelling the storage systems of TSO, the most recent version of ARM, and abstract specifications), or a storage system, $W$, formed from a list of "writes" to the global variables (modelling the storage systems of older versions of ARM and POWER). Hence a system is in one of the two following forms.

$$
\begin{array}{r}
\left(\operatorname{glb} \sigma \bullet\left(\operatorname{tid}_{1} \operatorname{lcl} \sigma_{1} \bullet c_{1}\right)\left\|\left(\operatorname{tid}_{2} \operatorname{lcl} \sigma_{2} \bullet c_{2}\right)\right\| \ldots\right) \\
\left(\operatorname{stg} W \bullet\left(\operatorname{tid}_{1} \operatorname{lcl} \sigma_{1} \bullet c_{1}\right)\left\|\left(\operatorname{tid}_{2} \operatorname{lcl} \sigma_{2} \bullet c_{2}\right)\right\| \ldots\right)
\end{array}
$$

\subsection{Operational semantics}

The meaning of our language is formalised using an operational semantics, summarised in Fig. 1. Given a program $c$ the operational semantics generates a trace, i.e., a possibly infinite sequence of steps $c_{0} \stackrel{\alpha_{1}}{\longrightarrow} c_{1} \stackrel{\alpha_{2}}{\longrightarrow} \ldots$ where the labels in the trace are actions, or a special label $\tau$ representing a silent or internal step that has no observable effect.

The terminated command nil has no behaviour; a trace that ends with this command is assumed to have completed. The effect of instruction prefixing in Rule (12) is discussed in Sect. 2.3. Note that actions become part of the trace. We describe an instantiation for reordering and forwarding corresponding to the semantics of ARM and POWER in Sect. 3.3 .

A nondeterministic choice (the internal choice of CSP [11]) can choose either branch, as given by Rule (13). The semantics of loops is given by unfolding, e.g., Rule (14) for a 'while' loop. Note that speculative execution, i.e., early execution of instructions which occur after a branch point 24, is theoretically unbounded, and loads from inside later iterations of the loop could occur in earlier iterations.

For ease of presentation in defining the semantics for local states, we give rules for specific forms of actions, i.e., assuming that $r$ is a local variable in the domain of $\sigma$, and that $x$ is a global (not in the domain of $\sigma$ ). The more general version can be straightforwardly constructed from the principles below. 


$$
(\alpha ; c) \stackrel{\alpha}{\longrightarrow} c \quad(a) \quad \begin{aligned}
& \frac{\beta}{\longrightarrow} c^{\prime} \alpha \stackrel{\mathrm{R}}{\models} \beta_{[\alpha]} \\
& (\alpha ; c) \stackrel{\beta_{[\alpha]}}{\longrightarrow}\left(\alpha ; c^{\prime}\right)
\end{aligned} \quad(b) \quad(12) \quad \begin{aligned}
& c \sqcap d \stackrel{\tau}{\longrightarrow} c \\
& c \sqcap d \stackrel{\tau}{\longrightarrow} d
\end{aligned}
$$

while $b$ do $c \stackrel{\tau}{\longrightarrow}([b] ; c$; while $b$ do $c) \sqcap([\neg b] ;$ nil $)$

$$
\begin{gathered}
\frac{c \stackrel{r:=v}{\longrightarrow} c^{\prime}}{(\mathbf{l} \mathbf{c l} \sigma \bullet c) \stackrel{\tau}{\longrightarrow}\left(\mathbf{l} \mathbf{c l} \sigma_{[r:=v]} \bullet c^{\prime}\right)} \\
\frac{c \stackrel{r:=x}{\longrightarrow} c^{\prime}}{(\mathbf{l c l} \sigma \bullet c) \stackrel{[x=v]}{\longrightarrow}\left(\mathbf{l c l} \sigma_{[r:=v]} \bullet c^{\prime}\right)}
\end{gathered}
$$

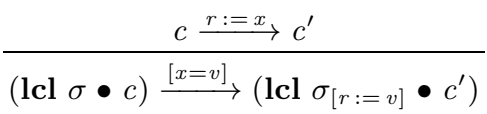

$$
\begin{aligned}
& \frac{p \stackrel{\alpha}{\longrightarrow} p^{\prime}}{\left(\operatorname{tid}_{\mathrm{N}} p\right) \stackrel{\mathrm{N}: \alpha}{\longrightarrow}\left(\operatorname{tid}_{\mathrm{N}} p^{\prime}\right)}(19) \\
& \frac{p \stackrel{\mathrm{N}: x:=e}{\longrightarrow} p^{\prime}}{(\operatorname{glb} \sigma \bullet p) \stackrel{\tau}{\longrightarrow}\left(\operatorname{glb} \sigma_{\left[x:=e_{\sigma}\right]} \bullet p^{\prime}\right)}
\end{aligned}
$$

$$
\frac{p_{1} \stackrel{\alpha}{\longrightarrow} p_{1}^{\prime}}{p_{1}\left\|p_{2} \stackrel{\alpha}{\longrightarrow} p_{1}^{\prime}\right\| p_{2}} \frac{p_{2} \stackrel{\alpha}{\longrightarrow} p_{2}^{\prime}}{p_{1}\left\|p_{2} \stackrel{\alpha}{\longrightarrow} p_{1}\right\| p_{2}^{\prime}}
$$

$$
\frac{p \stackrel{\mathrm{N}:[e]}{\longrightarrow} p^{\prime} \quad e_{\sigma} \equiv \text { true }}{(\text { glb } \sigma \bullet p) \stackrel{\tau}{\longrightarrow}\left(\text { glb } \sigma \bullet p^{\prime}\right)}
$$

Fig. 1. Semantics of the language

Rule (15) states that an action updating variable $r$ to value $v$ results in a change to the local state (denoted $\sigma_{[r:=v]}$ ). Since this is a purely local operation there is no interaction with the storage subsystem and hence the transition is promoted as a silent step $\tau$. Rule (16) states that a store of the value in variable $r$ to global $x$ is promoted as an instruction $x:=v$ where $v$ is the local value for $r$. Rule (17) covers the case of a load of $x$ into $r$. The value of $x$ is not known locally. The promoted label is a guard requiring that the value read for $x$ is $v$. This transition is possible for any value of $v$, but the correct value will be resolved when the label is promoted to the storage level. Rule (18) states that a guard is partially evaluated with respect to the local state before it is promoted to the global level. The notation $e_{\sigma}$ replaces $x$ with $v$ in $e$ for all $(x \mapsto v) \in \sigma$.

Rule (19) simply tags the process id to an instruction, to assist in the interaction with the storage system, and otherwise has no effect. Instructions of concurrent processes are interleaved in the usual way as described by Rule (20).

Other straightforward rules which we have omitted above include the promotion of fences through a local state, and that atomic sequences of actions are handled inductively by the above rules.

Multi-copy atomic storage subsystem. Traditionally, changes to shared variables occur on a shared global state, and when written to the global state are seen instantaneously by all processes in the system. This is referred to as multicopy atomicity and is a feature of TSO and the most recent version of ARM [21]. 
Older versions of ARM and POWER, however, lack such multi-copy atomicity and require a more complex semantics. We give the simpler case (covered in Fig. (1) first 4

Recall that at the global level the process id $\mathrm{N}$ has been tagged to the actions by Rule (19). Rule (21) covers a store of some expression $e$ to $x$. Since all local variable references have been replaced by their values at the process level due to Rules (15)-(18), expression $e$ must refer only to shared variables in $\sigma$. The value of $x$ is updated to the fully evaluated value, $e_{\sigma}$.

Rule (22) states that a guard transition $[e]$ is possible exactly when $e$ evaluates to true in the global state. If it does not, no transition is possible; this is how incorrect branches are eliminated from the traces. If a guard does not evaluate to true, execution stops in the sense that no transition is possible. This corresponds to a false guard, i.e., magic 2014, and such behaviours do not terminate and are ignored for the purposes of determining behaviour of a real system. Interestingly, this straightforward concept from standard refinement theory allows us to handle speculative execution straightforwardly. In existing approaches, the semantics is complicated by needing to restart reads if speculation proceeds down the wrong path. Treating branch points as guards works because speculation should have no effect if the wrong branch was chosen.

To understand how this approach to speculative execution works, consider the following derivation. Assume that (a) loads may be reordered before guards if they reference independent variables, and (b) loads may be reordered if they reference different variables. Recall that we omit trailing nil commands to save space.

$$
\begin{aligned}
& r_{1}:=x ;\left(\text { if } r_{1}=0 \text { then } \underline{\left.r_{2}:=y\right)}\right. \\
= & \text { Definition of if } \underline{100}) \\
& r_{1}:=x ;\left(\left(\left[r_{1}=0\right] ; \underline{\left.r_{2}:=y\right)} \sqcap\left[r_{1} \neq 0\right]\right)\right. \\
\sqsubseteq & \text { Resolve to the first branch, since }(c \sqcap d) \sqsubseteq c \\
& r_{1}:=x ;\left[r_{1}=0\right] ; \underline{r_{2}:=y} \\
\sqsubseteq & \text { From Law (7) and assumption (a) } \\
& r_{1}:=x ; \underline{r_{2}:=y} \cdot\left[r_{1}=0\right] \\
\sqsubseteq & \text { From Law (7) and assumption (b) } \\
& \underline{r_{2}:=y} \cdot r_{1}:=x ;\left[r_{1}=0\right]
\end{aligned}
$$

This shows that the inner load (underlined) may be reordered before the branch point, and subsequently before an earlier load. Note that this behaviour results in a terminating trace only if $r_{1}=0$ holds when the guard is evaluated, and otherwise becomes magic (speculation down an incorrect path). On ARM processors, placing a control fence (cfence) instruction inside the branch, before the inner load prevents this reordering (see Sect. 3.3).

Non-multi-copy atomic storage subsystem. Some versions of ARM and POWER allow processes to communicate values to each other without accessing

4 In this straightforward model of shared state there is no global effect of fences, and we omit the straightforward promotion rule. 


$$
\begin{aligned}
& p \stackrel{\mathrm{N}:[x=v]}{\longrightarrow} p^{\prime} \\
& \forall w \in \operatorname{ran}\left(W_{1}\right) \bullet x=w . v a r \Rightarrow \mathrm{N} \notin w . s e e n \\
& \left(\operatorname{stg} W_{1} \frown(x \mapsto v)_{\mathrm{NS}}^{\mathrm{M}} \frown W_{2} \bullet p\right) \stackrel{\mathrm{N}:[x=v]}{\longrightarrow}\left(\operatorname{stg} W_{1} \frown(x \mapsto v)_{\mathrm{NS} \cup\{\mathrm{N}\}}^{\mathrm{M}} \frown W_{2} \bullet p^{\prime}\right) \\
& p \stackrel{\mathrm{N}: x:=v}{\longrightarrow} p^{\prime} \\
& \forall w \in \operatorname{ran}\left(W_{1}\right) \bullet \mathrm{N} \neq w . \text { thread } \wedge(x=w . v a r \Rightarrow \mathrm{N} \notin w . \text { seen }) \\
& \left(\text { stg } W_{1} \frown W_{2} \bullet p\right) \stackrel{\mathrm{N}: x:=v}{\longrightarrow}\left(\operatorname{stg} W_{1} \frown(x \mapsto v)_{\{\mathrm{N}\}}^{\mathrm{N}} \frown W_{2} \bullet p^{\prime}\right) \\
& p \stackrel{\text { N:fence }}{\longrightarrow} p^{\prime} \\
& \overline{(\operatorname{stg} W \bullet p) \stackrel{\text { N:fence }}{\longrightarrow}\left(\operatorname{stg} f l u s h_{\mathrm{N}}(W) \bullet p^{\prime}\right)}
\end{aligned}
$$

where

$$
\text { flush }_{\mathrm{N}}(\langle\rangle)=\langle\rangle \quad \text { flush }_{\mathrm{N}}(w \frown W)= \begin{cases}w_{[\text {seen }:=\mathrm{PID}]} \frown \text { flush }_{\mathrm{N}}(W) & \text { if } \mathrm{N} \in \text { w.seen } \\ w \frown f l u s h_{\mathrm{N}}(W) & \text { otherwise }\end{cases}
$$

Fig. 2. Rules for the non-multi-copy atomic subsystem of ARM and POWER

the heap. That is, if process $p_{1}$ is storing $v$ to $x$, and process $p_{2}$ wants to load $x$ into $r, p_{2}$ may preemptively load the value $v$ into $r$, before $p_{1}$ 's store hits the global shared storage. Therefore different processes may have different views of the value of a global variable, as exposed by litmus tests such as the WRC family [3].

Our approach to modelling this is based on that of the operational model of 22 . However, that model maintains several partial orders on operations reflecting the nondeterminism in the system, whereas we let the nondeterminism be represented by choices in the operational rules. This means we maintain a simpler data structure, a single global list of writes. The shared state from the perspective of a given process is a particular view of this list. There is no single definitive shared state. In addition, viewing a value in the list causes the list to be updated and this affects later views. To obtain the value of a variable this list is searched starting with the most recent write first. A process $p_{1}$ that has already seen the latter of two updates to a variable $x$ may not subsequently then see the earlier update. Hence the list keeps track of which processes have seen which stores. Furthermore, accesses of the storage subsystem are influenced by fences.

A write $w$ has the syntactic form $(x \mapsto v)_{\mathrm{NS}}^{\mathrm{N}}$, where $x$ is a global variable being updated to value $v, \mathrm{~N}$ is the process id of the process from which the store originated, and NS is the set of process ids that have "seen" the write. For such a $w$, we let $w \cdot v a r=x, w$.thread $=\mathrm{N}$ and $w$.seen $=$ NS. For a write $(x \mapsto v)_{\mathrm{NS}}^{\mathrm{N}}$ it is always the case that $\mathrm{N} \in \mathrm{NS}$. The storage $W$ is a list of writes, 


$$
\begin{aligned}
& \alpha \stackrel{R}{\not} \text { fence } \quad(26) \quad x:=e \stackrel{\mathrm{R}}{\models} y:=f \text { iff } \\
& \text { fence } \stackrel{\text { R }}{*} \alpha \\
& \text { [b] } \beta \text { \& cfence } \\
& \text { cfence } \stackrel{\text { R }}{\not} r:=e \\
& {\left[b_{1}\right] \stackrel{\text { R }}{\models}\left[b_{2}\right]} \\
& {[b] \stackrel{R}{\&} \varphi:=e} \\
& \text { (30) } x:=e_{[y:=f]}=x:=e_{[y \backslash f]} \text { if } \\
& \begin{aligned}
x:=e \stackrel{\mathrm{R}}{\models} y & :=f \text { iff } \\
x & \neq y, x \text { nfi } f, y \text { nfi } e, \text { and }
\end{aligned} \\
& e, f \text { are load-distinct } \\
& x:=e \stackrel{\text { R }}{\Leftarrow}[b] \quad \text { iff } x \text { nfi } b \\
& \alpha \stackrel{\text { R }}{\Leftarrow} \beta \text { in all other cases } \\
& e \text { has no shared variables } \\
& {[e]_{[y:=f]}=\left[e_{[y \backslash f]}\right] \text { if }} \\
& e \text { has no shared variables } \\
& \beta_{[\alpha]}=\beta \text { otherwise }
\end{aligned}
$$

Fig. 3. Reordering and forwarding following ARM assembler semantics. Let $x, y$ denote any variable, $r$ a local variable, and $\varphi$ a global variable.

initially populated with writes for the initial values of global variables, which all processes have "seen".

We give two specialised rules (for a load and store) in Fig. [2 $2^{5}$ Rule (23) states that a previous write to $x$ may be seen by process $\mathrm{N}$ if there are no more recent writes to $x$ that it has already seen. Its id is added to the set of processes that have seen that write. Rule (24) states that a write to $x$ may be added to the system by process $\mathrm{N}$, appearing earlier than existing writes in the system, if the following two conditions hold for each of those existing writes $w$ : they are not by $\mathrm{N}(\mathrm{N} \neq w$.thread, local coherence), and $x=w \cdot v a r \Rightarrow \mathrm{N} \notin w$. seen, i.e., writes to the same variable are seen in a consistent order (although not all writes need be seen).

A fence action by process $\mathrm{N}$ 'flushes' all previous writes by and seen by $\mathrm{N}$. The flush function modifies $W$ so that all processes can see all writes by $\mathrm{N}$, effectively overwriting earlier writes. This is achieved by updating the write so that all processes have seen it, written as $w_{[\text {seen }:=P I D]}$.

\subsection{Reordering and forwarding for ARM and POWER}

Our general semantics is instantiated for ARM and POWER processors in Fig. 3 which provides particular definitions for the reordering relation and forwarding that are generalised from the orderings on stores and loads in these processors 6

5 To handle the general case of an assignment $x:=e$, where $e$ may contain more than one shared variable, the antecedents of the rules are combined, retrieving the value of each variable referenced in $e$ individually and accumulating the changes to $W$.

${ }^{6}$ We have excluded address shifting, which creates address dependencies [3, as this does not affect the majority of high-level algorithms in which we are interested. However, address dependencies are accounted for in our tool as discussed in Appendix B. 
Fences prevent all reorderings (26, 27). Control fences prevent speculative loads when placed between a guard and a load (28, 29). Guards may be reordered with other guards (30), but stores to shared variables may not come before a guard evaluation (31). This prevents speculative execution from modifying the global state, in the event that the speculation was down the wrong branch. An update of a local variable may be reordered before a guard provided it does not affect the guard expression (32). Guards may be reordered before updates if those updates do not affect the guard expression (33).

Assignments may be reordered as shown in (34) and discussed in Sect. 2.2. Forwarding is defined straightforwardly so that an earlier update modifies the expression of a later update or guard (35, 36), provided it references no shared variables.

\section{Model checking concurrent data structures}

Our semantics has been encoded in the Maude rewriting system [6]. We have used the resulting prototype tool to validate the semantics against litmus tests which have been used in other work on ARM (348 tests) 9] and POWER (758 tests) 22. As that research was developed through testing on hardware and in consultation with the processor vendors themselves we consider compliance with those litmus tests to be sufficient validation. With two exceptions, as discussed in Sect. 5, our semantics agrees with those results.

We have employed Maude as a model checker to verify that a (test-and-set) lock provides mutual exclusion on ARM and POWER, and that a lock-free stack algorithm, and a deque (double-ended queue) algorithm, satisfy their abstract specifications on ARM and POWER. We describe the verification of the deque below, in which we found a bug in the published algorithm.

\subsection{Chase-Lev deque}

Lê et. al [15] present a version of the Chase-Lev deque [5] adapted for ARM and POWER. The deque is implemented as an array, where elements may be put on or taken from the tail, and additionally, processes may steal an element from the head of the deque. The put and take operations may be executed by a single process only, hence there is no interference between these two operations (although instruction reordering could cause consecutive invocations to overlap). The steal operation can be executed by multiple processes concurrently.

The code we tested is given in Fig. 4 where $L$ is the maximum size of the deque which is implemented as a cyclic array, with all elements initialised to some irrelevant value. The original code includes handling array resizing, but here we focus on the insert/delete logic. For brevity we omit trailing nils. We have used a local variable return to model the return value, and correspondingly have refactored the algorithm to eliminate returns from within a branch. A $C A S(x, r, e)$ (compare-and-swap) instruction atomically compares the value of 


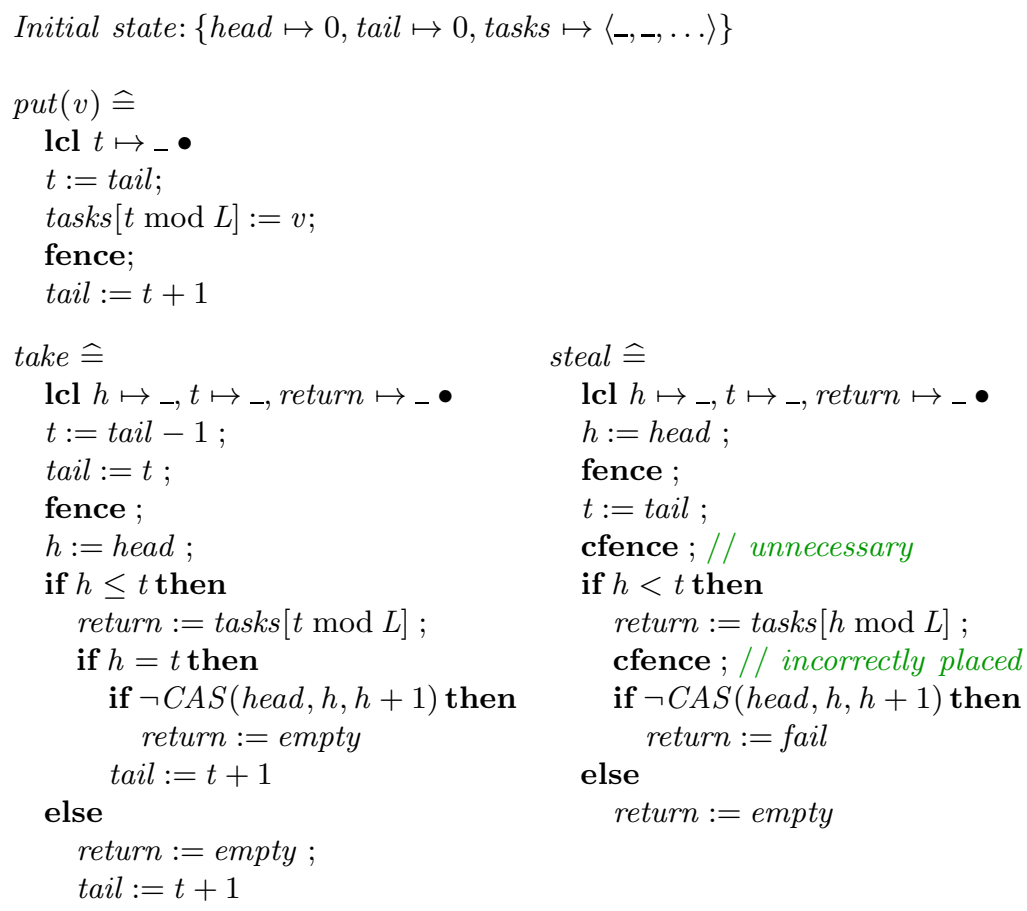

Fig. 4. A version of Lê et. al's work-stealing deque algorithm for ARM [15]

global $x$ with the value $r$ and if the same updates $x$ to $e$. We model a conditional statement with a $C A S$ as follows.

$$
\text { if } C A S(x, r, e) \text { then } c_{1} \text { else } c_{2} \widehat{=}\left(\langle[x=r], x:=e\rangle ; c_{1}\right) \sqcap\left([x \neq r] ; c_{2}\right)
$$

The put operation straightforwardly adds an element to the end of the deque, incrementing the tail index. It includes a full fence so that the tail pointer is not incremented before the element is placed in the array. The take operation uses a $C A S$ operation to atomically increment the head index. Interference can occur if there is a concurrent steal operation in progress, which also uses $C A S$ to increment head to remove an element from the head of the deque. The take and steal operation return empty if they observe an empty deque. In addition the steal operation may return the special value fail if interference on head occurs. Complexity arises if the deque has one element and there are concurrent processes trying to both take and steal that element at the same time.

Operations take and steal use a fence operation to ensure they have consistent readings for the head and tail indexes, and later use $C A S$ to atomically update the head pointer (only if necessary, in the case of take). Additionally, the steal operation contains two cfence barriers (ctrl_isync in ARM). 
Verification. We use an abstract model of the deque and its operations to specify the allowed final values of the deque and return values. The function last $(q)$ returns the last element in $q$ and $\operatorname{front}(q)$ returns $q$ excluding its last element.

$$
\begin{aligned}
& \operatorname{put}(v) \hat{=} q:=q \frown\langle v\rangle \\
& \text { take } \widehat{=} \mathbf{l c l} \text { return }:=\text { none } \bullet \\
& \langle[q=\langle\rangle], \text { return }:=\operatorname{empty}\rangle \sqcap \\
& \langle[q \neq\langle\rangle], \text { return }:=\operatorname{last}(q), q:=\operatorname{front}(q)\rangle \\
& \text { steal } \widehat{=} \mathbf{l c l} \text { return }:=\text { none } \\
& \langle[q=\langle\rangle], \text { return }:=\operatorname{empty}\rangle \sqcap \\
& \langle[q \neq\langle\rangle], \text { return }:=\operatorname{head}(q), q:=\operatorname{tail}(q)\rangle
\end{aligned}
$$

The abstract specification for steal does not attempt to detect interference and return fail. As such we exclude these behaviours of the concrete code from the analysis.

We model-checked combinations of one to three processes operating in parallel, each executing one or two operations in sequence. The final states of the abstract and concrete code were compared via a simulation relation. This exposed a bug in the code which may occur when a put and steal operation execute in parallel on an empty deque. The load return $:=\operatorname{tasks}[h \bmod L]$ can be speculatively executed before the guard $h<t$ is evaluated, and hence also before the load of tail. Thus the steal process may load head, load an irrelevant return value, at which point a put operation may complete, storing a value and incrementing tail. The steal operation resumes, loading the new value for tail and observing a non-empty deque, succeeding with its $C A S$ and returning the irrelevant value, which was loaded before the put operation had begun.

Swapping the order of the second cfence with the load of tasks $[h \bmod L]$ eliminates this bug, and our analysis did not reveal any other problems. In addition, eliminating the first cfence does not change the possible outcomes.

\section{Related work}

This work makes use of an extensive suite of tests elucidating the behaviour of weak memory models in ARM and POWER via both operational and axiomatic semantics $32217 / 9$. Those semantics were developed and validated through testing on real hardware and in consultation with processor vendors themselves. Our model is validated against their results, in the form of the results of litmus tests.

Excluding two tests involving "shadow registers", which appear to be processor-specific facilities which are not intended to conform to sequential semantics (they do not correspond to higher-level code), all of the 348 ARM litmus tests run on our model agreed with the results in [9], and all of the 758 POWER 
litmus tests run on our model agreed with the results in [22, which the exception of litmus test PP0015, which we give below, translated into our formal language 7

$$
\begin{aligned}
x:=1 ; & \text { fence } ; y:=1 \quad \\
r_{0}:=y ; & z:=\left(r_{0} \text { xor } r_{0}\right)+1 ; z:=2 ; r_{3}:=z ; \\
& \quad\left(\text { if } r_{3}=r_{3} \text { then nil else nil }\right) ; \text { cfence } ; r_{4}:=x
\end{aligned}
$$

The tested condition is $z=2 \wedge r_{0}=1 \wedge r_{4}=0$, which asks whether it is possible to load $x$ (the last statement of process 2) before loading $y$ (the first statement of process 2). At a first glance the control fence prevents the load of $x$ happening before the branch. However, as indicated by litmus tests such as MP+dmb.sy+fri-rfi-ctrisb, 9, Sect 3, Out of order execution], under some circumstances the branch condition can be evaluated early, as discussed in the speculative execution example. We expand on this below by manipulating the second process, taking the case where the success branch of the if statement is chosen. To aid clarity we underline the instruction that is the target of the (next) refinement step.

$$
r_{0}:=y ; z:=\left(r_{0} \text { xor } r_{0}\right)+1 ; z:=2 ; \underline{r_{3}:=z} ;\left[r_{3}=r_{3}\right] ; \text { cfence } ; r_{4}:=x
$$

$\sqsubseteq$ Promote load with forwarding (from $z:=2$ ), from Laws (6) and (7)

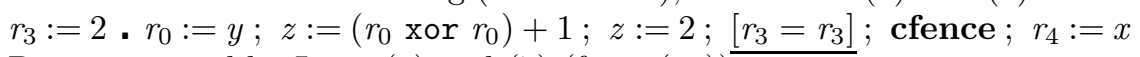

$\sqsubseteq$ Promote guard by Laws (6) and (77) (from (33))

$$
r_{3}:=2 \cdot\left[r_{3}=r_{3}\right] \cdot r_{0}:=y ; z:=\left(r_{0} \text { xor } r_{0}\right)+1 ; z:=2 ; \text { cfence } ; r_{4}:=x
$$

$\sqsubseteq$ Promote control fence by Laws (6) and (7) (28) does not now apply)

$$
r_{3}:=2 \text {. }\left[r_{3}=r_{3}\right] \text {. cfence } \cdot r_{0}:=y ; z:=\left(r_{0} \text { xor } r_{0}\right)+1 ; z:=2 ; \underline{r_{4}}:=x
$$

$\sqsubseteq$ Promote load by Laws (6) and (7)

$$
r_{3}:=2 \cdot\left[r_{3}=r_{3}\right] \text {. cfence } \cdot r_{4}:=x \cdot r_{0}:=y ; z:=\left(r_{0} \text { xor } r_{0}\right)+1 ; z:=2
$$

The load $r_{4}:=x$ has been reordered before the load $r_{0}:=y$, and hence when interleaved with the first process from (38) it is straightforward that the condition may be satisfied.

In the Flowing/POP model of [9], this behaviour is forbidden because there is a data dependency from the load of $y$ into $r_{0}$ to $r_{3}$, via $z$. This appears to be because of the consecutive stores to $z$, one of which depends on $r_{0}$. In the testing of real processors reported in 9 , the behaviour that we allow was never observed, but is allowed by the model in [3]. As such we deem this discrepancy to be a minor issue in Flowing/POP (preservation of transitive dependencies) rather than a fault in our model.

Our model of the storage subsystem is similar to that of the operational models of [229]. However our thread model is quite different, being defined in terms

\footnotetext{
7 We simplified some of the syntax for clarity, in particular introducing a higher-level if statement to model a jump command and implicit register (referenced by the compare (CMP) and branch-not-equal (BNE) instructions). We have also combined some commands, retaining dependencies, in a way that is not possible in the assembler language. The xor operator is exclusive-or; its use here artificially creates a data dependency [3] between the updates to $r_{0}$ and $z$.
} 
of relationships between actions. The key difference is how we handle branching and the effects of speculative execution. The earlier models are complicated in the sense that they are closer to the real execution of instructions on a processor, involving restarting reads if an earlier read invalidates the choice taken at a branch point.

The axiomatic models, as exemplified by [3], define relationships between instructions in a whole-system way, including relationships between instructions in concurrent processes. This gives a global view of how an architecture's reordering rules (and storage system) interact to reorder instructions in a system. Such global orderings are not immediately obvious from our pair-wise orderings on instructions. On the other hand, those globals orderings become quite complex and obscure some details, and it is unclear how to extract some of the generic principles such as (2).

\section{Conclusion}

We have utilised earlier work to devise a wide-spectrum language and semantics for weak memory models which is relatively straightforward to define and extend, and which lends itself to verifying low-level code against abstract specifications. While abstracting away from the details of the architecture, we believe it provides a complementary insight into why some reorderings are allowed, requiring a pairwise relationship between instructions rather than one that is system-wide.

A model-checking approach based on our semantics exposed a bug in an algorithm in 15 in relation to the placement of a control fence. The original paper includes a hand-written proof of the correctness of the algorithm based on the axiomatic model of [17]. The possible traces of the code were enumerated and validated against a set of conditions on adding and removing elements from the deque (rather than with respect to an abstract specification of the deque). The conditions being checked are non-trivial to express using final state analysis only. An advantage of having a semantics that can apply straightforwardly to abstract specifications, rather than a proof technique that analyses behaviours of the concrete code only, is that we may reason at a more abstract level.

We have described the ordering condition as syntactic constraints on atomic actions, which fits with the low level decisions of hardware processors such as ARM and POWER. However our main reordering principle (2) is based on semantic concerns, and as such may be applicable as a basis for understanding the interplay of software memory models, compiler optimisations and hardware memory models [14.

The wide-spectrum language has as its basic instruction an assignment, which is sufficient for specifying many concurrent programs. However we hope to extend the language to encompass more general constructs such as the specification command [19] and support rely-guarantee reasoning [12 13 10 17].

Acknowledgements We thank Kirsten Winter for feedback on this work, and the support of Australian Research Council Discovery Grant DP160102457. 


\section{References}

1. Sarita V. Adve and Hans-J. Boehm. Memory models: A case for rethinking parallel languages and hardware. Commun. ACM, 53(8):90-101, August 2010.

2. Jade Alglave, Luc Maranget, Susmit Sarkar, and Peter Sewell. Litmus: Running tests against hardware. In Parosh Aziz Abdulla and K. Rustan M. Leino, editors, Tools and Algorithms for the Construction and Analysis of Systems: 17th International Conference, TACAS 2011, Held as Part of the Joint European Conferences on Theory and Practice of Software, ETAPS 2011, Saarbrücken, Germany, March 26-April 3, 2011. Proceedings, pages 41-44, Berlin, Heidelberg, 2011. Springer Berlin Heidelberg.

3. Jade Alglave, Luc Maranget, and Michael Tautschnig. Herding cats: Modelling, simulation, testing, and data mining for weak memory. ACM Trans. Program. Lang. Syst., 36(2):7:1-7:74, July 2014.

4. R. J. Back and J. von Wright. Refinement Calculus: A Systematic Introduction. Springer-Verlag, 1998.

5. David Chase and Yossi Lev. Dynamic circular work-stealing deque. In SPAA'05: Proceedings of the 17th annual ACM symposium on Parallelism in algorithms and architectures, pages 21-28, New York, NY, USA, 2005. ACM Press.

6. Manuel Clavel, Francisco Duran, Steven Eker, Patrick Lincoln, Narciso Marti-Oliet, José Meseguer, and José F. Quesada. Maude: specification and programming in rewriting logic. Theoretical Computer Science, 285(2):187 - 243, 2002.

7. Robert J. Colvin, Ian J. Hayes, and Larissa A. Meinicke. Designing a semantic model for a wide-spectrum language with concurrency. Formal Aspects of Computing, 29(5):853-875, Sep 2017.

8. Edsger W. Dijkstra. Guarded commands, nondeterminacy and formal derivation of programs. Commun. ACM, 18(8):453-457, August 1975.

9. Shaked Flur, Kathryn E. Gray, Christopher Pulte, Susmit Sarkar, Ali Sezgin, Luc Maranget, Will Deacon, and Peter Sewell. Modelling the ARMv8 architecture, operationally: Concurrency and ISA. In Proceedings of the 43rd Annual ACM SIGPLAN-SIGACT Symposium on Principles of Programming Languages, POPL '16, pages 608-621, New York, NY, USA, 2016. ACM.

10. Ian J. Hayes, Robert J. Colvin, Larissa A. Meinicke, Kirsten Winter, and Andrius Velykis. An algebra of synchronous atomic steps. In John Fitzgerald, Constance Heitmeyer, Stefania Gnesi, and Anna Philippou, editors, FM 2016: Formal Methods: 21st International Symposium, Limassol, Cyprus, November 9-11, 2016, Proceedings, pages 352-369, Cham, 2016. Springer International Publishing.

11. C. A. R. Hoare. Communicating Sequential Processes. Prentice-Hall, Inc., Upper Saddle River, NJ, USA, 1985.

12. Cliff B. Jones. Specification and design of (parallel) programs. In IFIP Congress, pages 321-332, 1983.

13. Cliff B. Jones. Tentative steps toward a development method for interfering programs. ACM Trans. Program. Lang. Syst., 5:596-619, October 1983.

14. Jeehoon Kang, Chung-Kil Hur, Ori Lahav, Viktor Vafeiadis, and Derek Dreyer. A promising semantics for relaxed-memory concurrency. In Proceedings of the 44th ACM SIGPLAN Symposium on Principles of Programming Languages, POPL 2017, pages 175-189, New York, NY, USA, 2017. ACM.

15. Nhat Minh Lê, Antoniu Pop, Albert Cohen, and Francesco Zappa Nardelli. Correct and efficient work-stealing for weak memory models. In Proceedings of the 18th ACM SIGPLAN Symposium on Principles and Practice of Parallel Programming, PPoPP '13, pages 69-80, New York, NY, USA, 2013. ACM. 
16. Sela Mador-Haim, Rajeev Alur, and Milo M. K. Martin. Generating litmus tests for contrasting memory consistency models. In Tayssir Touili, Byron Cook, and Paul Jackson, editors, Computer Aided Verification: 22nd International Conference, CAV 2010, Edinburgh, UK, July 15-19, 2010. Proceedings, pages 273-287, Berlin, Heidelberg, 2010. Springer Berlin Heidelberg.

17. Sela Mador-Haim, Luc Maranget, Susmit Sarkar, Kayvan Memarian, Jade Alglave, Scott Owens, Rajeev Alur, Milo M. K. Martin, Peter Sewell, and Derek Williams. An axiomatic memory model for POWER multiprocessors. In Proceedings of the 24th International Conference on Computer Aided Verification, CAV'12, pages 495-512, Berlin, Heidelberg, 2012. Springer-Verlag.

18. Robin Milner. A Calculus of Communicating Systems. Springer-Verlag New York, Inc., 1982.

19. Carroll Morgan. The specification statement. ACM Trans. Program. Lang. Syst., 10:403-419, July 1988.

20. Carroll Morgan. Programming from Specifications. Prentice Hall, second edition, 1994.

21. Christopher Pulte, Shaked Flur, Will Deacon, Jon French, Susmit Sarkar, and Peter Sewell. Simplifying ARM concurrency: Multicopy-atomic axiomatic and operational models for ARMv8. In Proceedings of the ACM SIGPLAN-SIGACT Symposium on Principles of Programming Languages (POPL). ACM Press, 2018. To appear.

22. Susmit Sarkar, Peter Sewell, Jade Alglave, Luc Maranget, and Derek Williams. Understanding POWER multiprocessors. SIGPLAN Not., 46(6):175-186, June 2011.

23. Peter Sewell, Susmit Sarkar, Scott Owens, Francesco Zappa Nardelli, and Magnus O. Myreen. X86-TSO: A rigorous and usable programmer's model for x86 multiprocessors. Commun. ACM, 53(7):89-97, July 2010.

24. Daniel J. Sorin, Mark D. Hill, and David A. Wood. A Primer on Memory Consistency and Cache Coherence. Morgan \& Claypool Publishers, 1st edition, 2011. 


\section{A Lightweight fences}

POWER's lightweight fences maintain order between loads, loads then stores, and stores, but not stores and subsequent loads (loads can come before earlier stores). If lightweight fences did not maintain load-load order it would be straightforward to define their effect in terms of one instruction. However to allow reordering later loads with earlier stores but not earlier loads we model a lwfence as two "gates", one blocking loads and one stores.

We define lwfence; $c$ as fence $_{L} ;$ fence $_{S} ; c$ where

$$
\begin{aligned}
& r:=x \stackrel{\text { R }}{\not} \text { fence }_{L} \quad \text { fence }_{L} \stackrel{R}{\not}=r:=x \\
& x:=v \& \text { fence }_{S} \quad \text { fence }_{S} \& x:=v
\end{aligned}
$$

Consider the code $c$; fence $_{L}$; fence fen $d$ for arbitrary $c$ and $d$. Without the intervening gates that constitute a lightweight fence the instructions of $c$ and $d$ could be reordered according to the usual restrictions. The fence ${ }_{S}$ instruction however prevents stores in $d$ from interleaving with instructions in $c$ (but loads may be reordered past the fence $_{S}$ instruction). Additionally, the fence f $_{L}$ may be reordered past any stores in $c$ but not past any loads. Hence between the two fence instructions can mix stores from $c$ and loads from $d$, which may be reordered subject to the usual constraints. The lightweight fence therefore maintains store/store, load/load, and load/store order.

A lightweight fence also has a global effect on the storage system, which

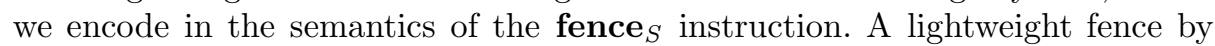
$\mathrm{N}$ marks any store in $W$ seen by $\mathrm{N}$ with a tag $\operatorname{Iwf}(\mathrm{N})$. A store $w$ by process $\mathrm{N}$ may not be inserted in $W$ before a write with the $\operatorname{Iwf}(\mathrm{N})$ tag. In addition, if another process $\mathrm{M}$ loads a value stored by $\mathrm{N}$, it sees not only that store but also all stores marked with $\operatorname{Iwf}(\mathrm{N})$. This transitive effect gives cumulativity of lightweight fences [3].

As fence f $_{S}$ is the latter to reach the storage system we give it the global

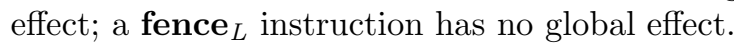

$$
\frac{p \stackrel{\text { N:fence }}{\longrightarrow} p^{\prime}}{(\text { stg } W \bullet p) \stackrel{\text { N:fence }}{\longrightarrow}\left(\text { stg } l w f l u s h_{\mathrm{N}}(W) \bullet p^{\prime}\right)}
$$

where

$$
\begin{aligned}
& \text { lwflush }_{\mathrm{N}}(\langle\rangle)=\langle\rangle
\end{aligned}
$$

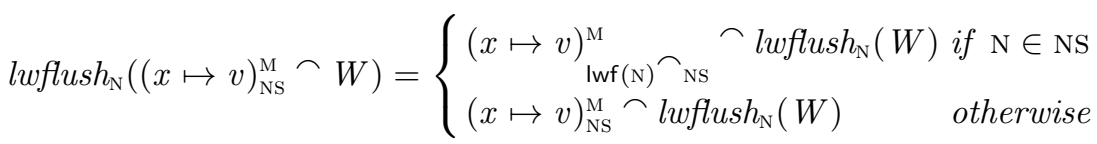

Adding the $\operatorname{lwf}(\mathrm{N})$ tag to the list of process ids that have observed a write affects the allowed ordering of how writes are seen. The key point is that $\mathrm{N}$ now sees, and lightweight-fences, writes by M that M has lightweight-fenced. 
The antecedent for Rule (24) needs to be updated to include $\operatorname{Iwf}(\mathrm{N}) \notin w$.seen as a further constraint on where writes can be placed in the global order $W$ : a write may not come before a write that the process has lightweight-fenced, even if that write is to a different variable.

\section{B Address shifting}

In ARM (and POWER) the value loaded from (or stored to) an address may be shifted. For the majority of high-level algorithms such details are hidden. However address shifting is investigated at the hardware level because it can affect reordering - so called "address dependencies" [3. The instruction LDR R1, [R2, X] loads into R1 the value at address X shifted by the amount in R2. To precisely model the semantics of address shifting requires a more concrete model than the one we propose, however, as determined by the litmus tests of [9], the effects of address dependencies can be investigated even when the shift amount is 0 (resulting in a load of the value at the address). As such we define that an address shift of 0 on a variable $x$ gives $x$, and leave the effect of other shift amounts undefined.

Address dependencies constrain the reorderings in the following ways: a branch may not be reordered before a load or store with an (unresolved) address dependency; a store may not be reordered before an instruction with an (unresolved) address dependency; and any instruction $\alpha$ which shares a register or variable with $\beta$ where $\beta$ has an unresolved address dependency may not be reordered with $\beta$. We incorporate these conditions into the general rule for assignments.

A further consequence of address shifting is that a load $r_{2}:=x$ may be reordered before $r_{1}:=[n, x]$ even though this would violate load-distinctness. However, to preserve coherence-per-location, the load into $r_{2}$ must not load a value of $x$ that was written before the value read by the load into $r_{1}$. This complex situation is handled in 9 by restarting load instructions if an earlier value is read into $r_{2}$. We handle it more abstractly by treating the load as speculation, where if an earlier value for $r_{2}$ is loaded then the effect of that speculation is thrown away.

We can give this extra semantics by adding an extra operational rule which applies only in those specific circumstances.

$$
\frac{p \stackrel{r_{2}:=x}{\longrightarrow} p^{\prime}}{r_{1}:=[n, x] ; p \stackrel{r_{2}:=x}{\longrightarrow} r_{1}:=[n, x] ;\left[r_{1}=r_{2}\right] ; p^{\prime}}
$$

In practical terms it is possible the first load of $x$ (into $r_{1}$ ) is delayed while determining the offset value. The later load is allowed to proceed, freeing up $p^{\prime}$ to continue speculatively executing until the dependency is resolved. The load into $r_{1}$ then must still be issued, the result being checked against $r_{2}$. This check must occur as to preserve coherency as the load into $r_{2}$ cannot read a value earlier than that read into $r_{1}$. Note that loads in $p^{\prime}$ can now potentially be reordered to execute ahead of the load into $r_{1}$. 